\title{
A Detail Study of Wavelet Families for EMG Pattern Recognition
}

\author{
Jingwei Too', A. R. Abdullah², Norhashimah Mohd Saad ${ }^{3}$, N Mohd Ali ${ }^{4}, \mathrm{H} \mathrm{Musa}^{5}$ \\ ${ }^{1,2,4}$ Fakulti Kejuruteraan Elektrik, Universiti Teknikal Malaysia Melaka, Malaysia \\ ${ }^{2,3}$ Centre of Excellence in Robotic and Industrial Automation, Universiti Teknikal Malaysia Melaka, Malaysia \\ ${ }^{3}$ Fakulti Kejuruteraan Elektronik dan Kejuruteraan Komputer, Universiti Teknikal Malaysia Melaka, Malaysia \\ ${ }^{5}$ Fakulti Pengurusan Teknologi dan Teknousahawanan Technology, Universiti Teknikal Malaysia Melaka, Malaysia
}

\begin{tabular}{|c|c|}
\hline Article Info & ABSTRACT \\
\hline Article history: & Wavelet transform (WT) has recently drawn the attention of the researchers \\
\hline Received Feb 23, 2018 & However, the optimal mother wavelet selection remains a challenge to the \\
\hline Revised Jul 19, 2018 & application of WT in EMG signal processing. This paper presents a detail \\
\hline Accepted Aug 7, 2018 & $\begin{array}{l}\text { study for different mother wavelet function in discrete wavelet transform } \\
\text { (DWT) and continuous wavelet transform (CWT). Additionally, the }\end{array}$ \\
\hline Keyword: & $\begin{array}{l}\text { performance of different mother wavelet in DWT and CWT at different } \\
\text { decomposition level and scale are also investigated. The mean absolute value }\end{array}$ \\
\hline Continuous w & (MAV) and wavelength (WL) features are extracted from each CWT and \\
\hline Discrete wavelet transform & $\begin{array}{l}\text { reconstructed DWT wavelet coefficient. A popular machine learning method, } \\
\text { support vector machine (SVM) is emploved to classify the different types of }\end{array}$ \\
\hline Electromyography & hand movements. The results showed that the most suitable mother wavelet \\
\hline Mother wavelet & in CWT are Mexican hat and Symlet 6 at scale 16 and 32, respectively. On \\
\hline Pattern recognition & $\begin{array}{l}\text { the other hand, Symlet } 4 \text { and Daubechies } 4 \text { at the second decomposition level } \\
\text { are found to be the optimal wavelet in DWT. From the analysis, we deduced } \\
\text { that Symlet } 4 \text { at the second decomposition level in DWT is the most suitable } \\
\text { mother wavelet for accurate classification of EMG signals of different hand } \\
\text { movements. }\end{array}$ \\
\hline
\end{tabular}

Copyright $@ 2018$ Institute of Advanced Engineering and Science. All rights reserved.

\section{Corresponding Author:}

Jingwei Too,

Faculty of Electrical Engineering,

Universiti Teknikal Malaysia Melaka,

Hang Tuah Jaya, 76100 Durian Tunggal, Melaka, Malaysia.

Email: jamesjames868@gmail.com

\section{INTRODUCTION}

Electromyography (EMG) signal contains rich muscle information that can be used in clinical and rehabilitation application. The potential of EMG signal in myoelectric control has been widespread since last two decades [1]. EMG signal recorded from a contracting muscle not only measures the time detection of muscle activation but also provides electrical signs of muscular behavior [2]. Recently, the analysis of EMG signal using a powerful signal processing technique has become the attention of the researchers.

In biomedical signal processing, short time Fourier Transform (STFT), wavelet transform (WT) and empirical decomposition mode (EMD) are frequently used [3]-[5]. In the previous research, it has been found that WT outperformed other time-frequency methods in discriminating EMG patterns [3],[6]. WT exhibits good time resolution at high frequency and good frequency resolution at low frequency components [7]. In general, WT can be categorized into discrete and continuous form. In continuous wavelet transform (CWT), the wavelet transformation changes continuously. On one side, discrete wavelet transform (DWT) decomposes the signal into multiresolution coefficients using high pass and low pass filters. 
Most studies to date indicated the performances of CWT and DWT were depending on the selection of a mother wavelet function [3],[8]-[10]. In the past studies, Kakoty et al. [8] investigated the best mother wavelet in DWT and CWT at different scale and decomposition level. The authors recommended the Gaussian and Symlet 8 to be the optimal mother wavelets in CWT and DWT, respectively. Phinyomark et al. [11] suggested that the use of DWT with the Daubechies 7 and 8 to ensure higher classification accuracy. Omari et al. [6] studied four mother wavelet functions at four different decomposition levels. The authors reported Symlet 4 offered the low classification error rate. Previous studies showed that the analysis of best mother wavelet in WT is critically important, leading to the optimum classification performance. However, the selection of mother wavelet is remains challenging in many areas.

The best mother wavelet is mostly subject independent, which means different mother wavelet offers different kind of performance on different subject. In addition, previous works mostly focus on four to eight mother wavelets in the classification of EMG signals, which is insufficient. Moreover, the performance of mother wavelet at different scale and decomposition level provide significant difference in classification performance. It is obvious that the analysis of the mother wavelet in CWT and DWT is remain insufficient and unclear in EMG pattern recognition. Therefore, this study aims to evaluate the best mother wavelet in CWT and DWT by employing a large number of mother wavelet functions with different scale and decomposition level, respectively.

This paper presents a detail study of the selection of mother wavelet in DWT and CWT. 14 mother wavelets of DWT and 12 mother wavelets of CWT at three different decomposition levels and scales are investigated, respectively. Two popular features mean absolute value (MAV) and wavelength (WL) are extracted from each wavelet coefficient for performance evaluation. The multiclass support vector machine (SVM) is used to classify EMG signal since it offers better performance in previous work [8],[12]. Finally, the best mother wavelet of DWT and CWT that offer the best classification performance are pointed.

\section{MATERIAL AND RESEARCH METHOD}

\subsection{EMG data collection}

This study was performed on ten healthy subjects ( 8 males and 2 females) with mean age of 28.6 $(\boldsymbol{\sigma}=9.7)$ years. Each subject provided informed consent to participate in the experiment. Additionally, all subjects were free from neurological and muscular disorder. Two wearable EMG devices named Shimmer (Shimmer3 Consensys EMG Development Kits) with standard setting were used in data collection. The resolution was set at 24 bits with a gain of 12. The EMG signal was gathered from four useful hand muscles namely extensor digitorum (ch1), flexor carpi radialis (ch2), extensor carpi radialis longus (ch3) and flexor carpi ulnaris (ch4) with two reference electrodes at the elbow. The signal was sampled at $1024 \mathrm{~Hz}$ and bandpass filtered between 20 and $500 \mathrm{~Hz}$. The skin was shaved and cleaned with alcohol pad before the electrode placement. The surface electrodes with $30 \mathrm{~mm}$ diameter were used and the inter-electrode distance was set at $20 \mathrm{~mm}$ to reduce the crosstalk. The bipolar electrode configuration was shown in Figure 1.

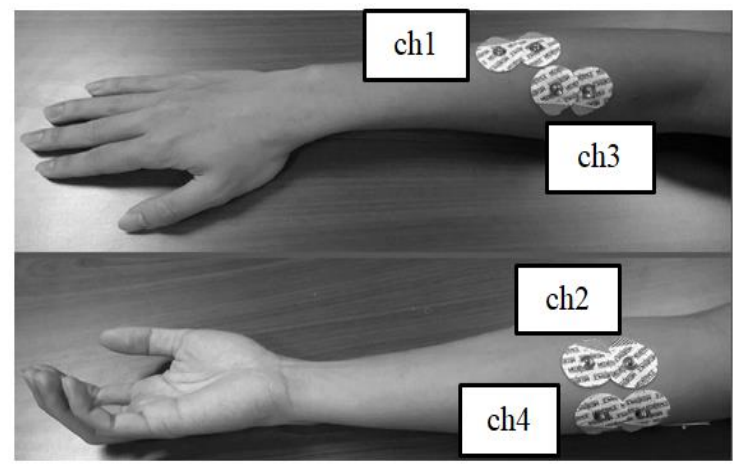

Figure 1. Electrodes configuration

Subject was seated comfortably on a chair with the hand in neutral position. The surface EMG signals were recorded as the subject performed ten different hand movements including thumb flexion (M1), thumb extension (M2), wrist flexion (M3), wrist extension (M4), making a fist (M5), pinch index to the thumb (M6), pinch middle to the thumb (M7), pinch ring to the thumb (M8), pinch little to the thumb (M9) 
and rest (M10). The experiments consisted of ten trials. Within each trial, the subject was asked to perform ten different hand movements for $5 \mathrm{~s}$ each, followed by a resting state of $4 \mathrm{~s}$. Moreover, a resting period of 1 min was introduced at the end of trial to avoid mental and muscle fatigue. The resting state was removed before data segmentation.

A recent report of real time EMG application indicated that the optimal window length was ranging from 150 to $250 \mathrm{~ms}$ to balance the controller delay and classification error rate [13]. Additionally, an overlapped windowing technique was introduced to produce better classification accuracy in EMG pattern recognition [14]. In this work, the EMG data were divided into $250 \mathrm{~ms}$ window (256 samples) with $50 \%$ (128 samples) overlapped. In total, a data matrix of 39 segments $\times 256$ samples $\times 4$ channels were obtained from each movement from each subject.

Figure 2 shows the flow diagram of the proposed recognition system. In the first stage, the raw EMG signals are collected and segmented. Next, MAV and WL features are extracted from CWT and reconstructed DWT coefficients at different scale and decomposition level using different mother wavelet, respectively. In the final stage, the SVM is used to recognize the EMG signals of ten different hand movements.

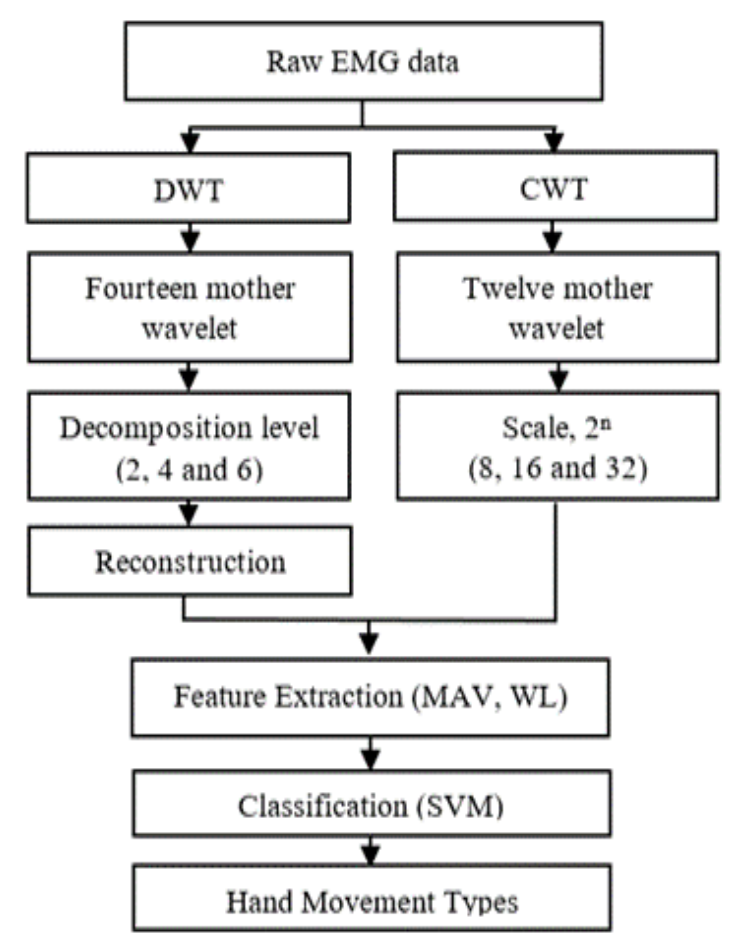

Figure 2. The flow diagram of the proposed recognition system

\subsection{Wavelet Transform}

Wavelet transform (WT) is a powerful mathematical tool that is successful in the analysis of biosignal including EMG signal. WT offers high frequency resolution for low frequency component and good time resolution for the high frequency component [13]. Generally, WT can be categorized into continuous and discrete forms. Continuous wavelet transform (CWT) decomposes the signal based on the dilations and translations of a single mother wavelet function. CWT is more consistent and efficient because it provides localization time-frequency information without down-sampling [11]. Additionally, CWT is continuous in term of shifting and it gives useful time-frequency information [15]. CWT can be defined as:

$$
C W T_{x}(\mathrm{~s}, b)=\int x(t) \psi_{\mathrm{s}, b}(t) d t
$$

where $x(t)$ is the input signal and $\psi_{s, b}(t)$ is the transformation of the mother wavelet function. The transformation can be expressed as: 


$$
\psi_{\mathrm{s}, b}(t)=\frac{1}{\sqrt{s}} \psi\left(\frac{t-b}{s}\right)
$$

where $s$ is the scaling parameter, $b$ is referred to the translation parameter and $\Psi(t)$ is called mother wavelet. The variables $s$ and $b$ provide the time scaling and shifting operation, respectively [16]. By using equation 1 and 2, CWT can be computed as:

$$
C W T(\mathrm{~s}, b)=\frac{1}{\sqrt{s}} \int_{-\infty}^{\infty} x(t) \psi\left(\frac{t-b}{s}\right) d t
$$

Figure 3 demonstrates the scalogram of CWT at scale 32 using Mexican hat wavelet. The yellow areas represent higher amplitude at each scale. In turn, dark blue areas refer to low amplitude.

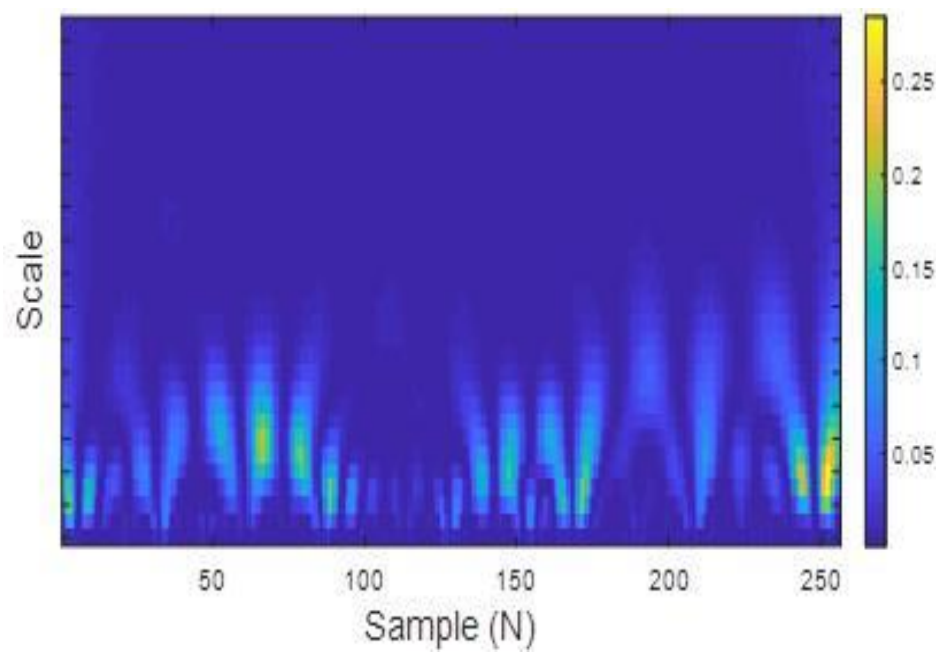

Figure 3. Scalogram of continuous wavelet transform at scale 32 using Mexican hat

Discrete wavelet transform (DWT) is derived from CWT [17]. DWT is more widely used because it offers low computation cost [11]. In DWT, the signal is decomposed into the approximation and detail coefficient which involves the change of sampling rate [18]. The decomposition of DWT comprises of two digital filters, which are high-pass and low-pass filters. The low-pass and high-pass filter down-sample the input signal and provide the approximation, $A$ and detail, $D$, respectively [11],[19]. For each decomposition level, the filters down-sample the signal by the factor of 2 . The first level of decomposition is defined as:

$$
\begin{aligned}
& \mathrm{D}[n]=\sum_{n} x[\mathrm{k}] \cdot h[2 n-k] \\
& \mathrm{A}[\mathrm{n}]=\sum_{n} x[\mathrm{k}] \cdot g[2 n-k]
\end{aligned}
$$

where $x[k]$ is the input signal, $D[n]$ is referred to the detail, $D_{l}$ and $A[n]$ is the approximation, $A_{l}$. The decomposition process is repeated until the desired final level is achieved. In the previous research, each coefficient subset was reconstructed to obtain more reliable EMG signal part, resulting in better classification accuracy [3],[13]. Therefore, the inverse wavelet transform is used to reconstruct each wavelet coefficient into more effective subset, namely, estimated approximation, $r A$ and estimated detail, $r D$. For example, the estimated subset $r D_{3}$ is obtained by performing the inverse wavelet transform on third-level detail, $D_{3}$. The wavelet reconstruction of estimated detail $\left(r D_{1}-r D_{6}\right)$ and estimated approximation $\left(r A_{1}-r A_{6}\right)$ were shown in Figure 4. 

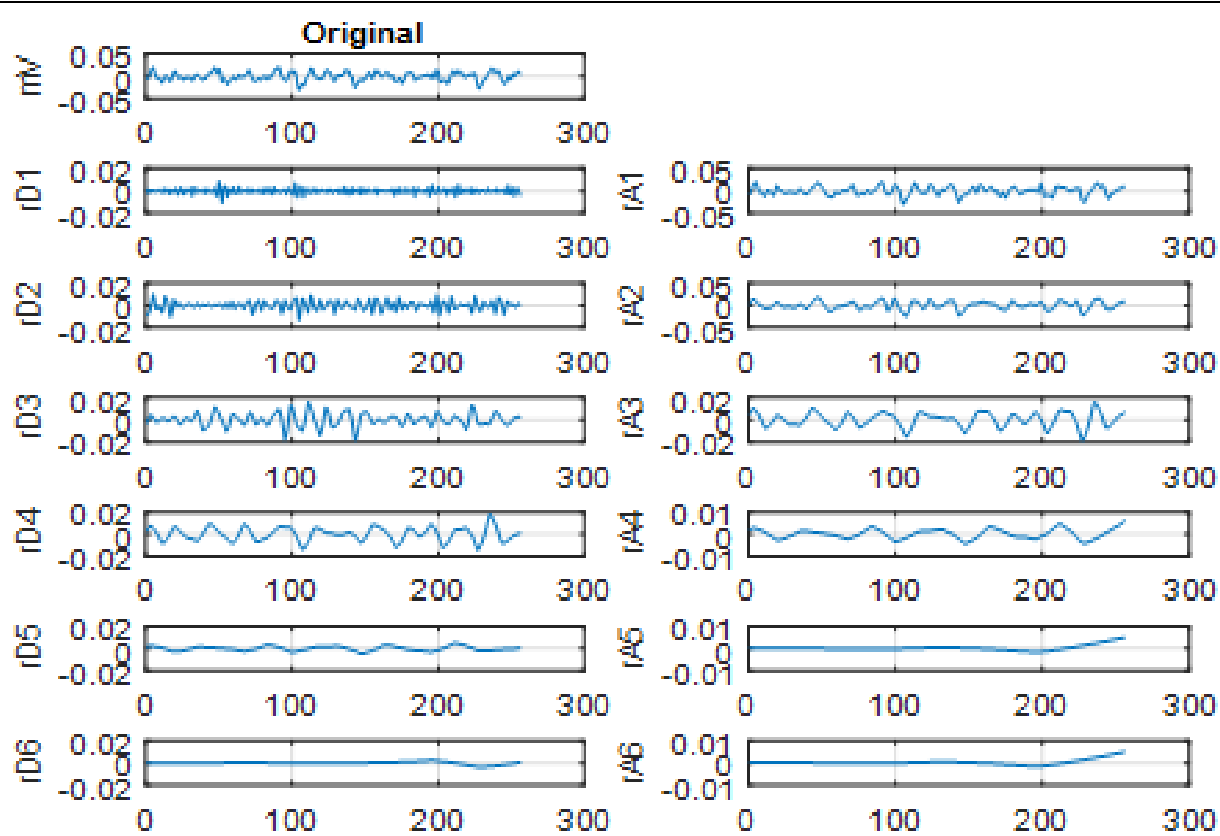

Figure 4. Wavelet reconstruction of DWT at sixth decomposition level using Symlet 4

\subsection{Mother Wavelet Selection and Evaluation}

Recent studies indicated WT has been recognized as one of the best time-frequency method in biomedical signal processing [3],[18],[20]. However, the performance of WT is mostly depending on the mother wavelet function. The selection of mother wavelet is remained challenging in many areas. Therefore, this work aims to evaluate the best mother wavelet in DWT and CWT for EMG signal processing. In this study, 14 mother wavelets in DWT and 12 mother wavelets in CWT are investigated. Table 1 is a lookup table of the mother wavelet used in CWT and DWT. It is worth noting different scale and decomposition level in CWT and DWT provide different property. For this reason, the performance of the mother wavelet at the scale $8,16,32$ and decomposition level of 2, 4 and 6 are examined.

\begin{tabular}{|c|c|c|}
\hline & CWT & DWT \\
\hline 1 & Haar & Haar \\
\hline 2 & Daubechies 2 & Daubechies 2 \\
\hline 3 & Daubechies 4 & Daubechies 4 \\
\hline 4 & Daubechies 6 & Daubechies 6 \\
\hline 5 & Symlet 2 & Daubechies 8 \\
\hline 6 & Symlet 4 & Daubechies 10 \\
\hline 7 & Symlet 6 & Symlet 2 \\
\hline 8 & Morlet & Symlet 4 \\
\hline 9 & Mayer & Symlet 6 \\
\hline 10 & Mexicanhat & Symlet 8 \\
\hline 11 & Gaussian 2 & Coiflet 2 \\
\hline 12 & Gaussian 4 & Coiflet 3 \\
\hline 13 & - & Coiflet 4 \\
\hline 14 & - & Coiflet 5 \\
\hline
\end{tabular}

\subsection{Feature Extraction using Wavelet Transform}

Feature extraction is an essential step to reduce the dimensionality and extract the useful information from the signal. In this work, wavelength (WL) and mean absolute value (MAV) are extracted from each wavelet coefficient. MAV and WL can be expressed as [6]:

$$
M A V=\frac{1}{L} \sum_{n=1}^{L}\left|x_{n}\right|
$$




$$
W L=\sum_{n=1}^{L-1}\left|x_{n+1}-x_{n}\right|
$$

where $X_{n}$ is the input signal and $L$ is the length of signal.

\subsection{Support Vector Machine}

Support vector machine (SVM) has been proved to be an outstanding supervised machine learning method in EMG pattern recognition [14]. Moreover, SVM has shown its superiority, especially for non-linear and high dimensional pattern recognition [21]. SVM maps the predictors onto a high dimensional space by using the concept of hyperplane partition for the data [22]. Some drawbacks of SVM are the complexity of the selection of kernel function and the longer computation time [14]. A previous study reported that radial basis function (RBF) was the best kernel function because it gave a higher classification performance [6]. In this regard, SVM with RBF kernel function is applied and it can be defined as:

$$
K\left(x, x_{i}\right)=\exp \left(-\frac{\left\|x-x_{i}\right\|^{2}}{2 \sigma^{2}}\right)
$$

where $x-x_{i}$ is the Euclidean distance between feature vectors and $\sigma$ is the kernel parameter.

\section{RESULTS AND ANALYSIS}

In this work, 10-fold cross validation is applied in the classification of EMG signals. The data is separated into 10 equal parts. Every part takes turn to test and the remaining parts are used in training phase. In the first part of the experiments, 14 mother wavelet functions in DWT at the three different decomposition level are evaluated. Table 2 outlines the mean classification accuracy of 14 mother wavelets of DWT at a decomposition level of 2, 4 and 6 across ten different subjects. From the results, the mean classification accuracy is found to be above $97 \%$ for all 14 mother wavelet functions in both WL and MAV feature sets. Additionally, MAV has shown to be an effective and reliable feature because it offers better performance in discriminating EMG patterns. By employing MAV feature, it is obvious that the highest classification accuracy is obtained by Symlet 4 (98.74\%), followed by Daubechies 4 (98.72\%) at the second decomposition level. On the other hand, Coiflet 3 outperforms other mother wavelets with a mean classification accuracy of 98.49\% at the fourth decomposition level when WL is used. From the analysis, Symlet 4 and Daubechies 4 at the second decomposition level are found to be the most suitable mother wavelet in DWT.

Table 2. Classification Accuracy (mean \pm STD) of 14 Mother Wavelets of DWT at Three Different

\begin{tabular}{|c|c|c|c|c|c|c|c|}
\hline \multirow{2}{*}{\multicolumn{2}{|c|}{ Mother wavelet }} & \multicolumn{2}{|c|}{ Classification performance (\%) } & \multirow{2}{*}{\multicolumn{2}{|c|}{ Mother wavelet }} & \multicolumn{2}{|c|}{ Classification performance (\%) } \\
\hline & & WL & MAV & & & WL & MAV \\
\hline \multirow{3}{*}{ Haar } & Level 2 & $97.90 \pm 1.02$ & $98.43 \pm 0.88$ & Sym 4 & Level 2 & $98.09 \pm 0.92$ & $98.74 \pm 0.66$ \\
\hline & Level 4 & $98.00 \pm 0.90$ & $98.28 \pm 0.80$ & & Level 4 & $98.36 \pm 0.78$ & $98.53 \pm 0.67$ \\
\hline & Level 6 & $97.28 \pm 0.94$ & $97.64 \pm 0.85$ & & Level 6 & $97.50 \pm 0.79$ & $97.67 \pm 0.81$ \\
\hline \multirow{3}{*}{$\mathrm{Db} 2$} & Level 2 & $97.97 \pm 1.01$ & $98.63 \pm 0.68$ & Sym 6 & Level 2 & $98.18 \pm 0.87$ & $98.67 \pm 0.76$ \\
\hline & Level 4 & $98.31 \pm 0.72$ & $98.44 \pm 0.70$ & & Level 4 & $98.39 \pm 0.67$ & $98.55 \pm 0.68$ \\
\hline & Level 6 & $97.32 \pm 0.94$ & $97.45 \pm 0.78$ & & Level 6 & $97.58 \pm 0.84$ & $97.65 \pm 0.87$ \\
\hline \multirow{3}{*}{$\mathrm{Db} 4$} & Level 2 & $98.08 \pm 0.88$ & $98.72 \pm 0.67$ & Sym 8 & Level 2 & $98.19 \pm 0.87$ & $98.70 \pm 0.71$ \\
\hline & Level 4 & $98.36 \pm 0.78$ & $98.56 \pm 0.68$ & & Level 4 & $98.45 \pm 0.69$ & $98.57 \pm 0.72$ \\
\hline & Level 6 & $97.36 \pm 0.91$ & $97.55 \pm 0.82$ & & Level 6 & $97.67 \pm 0.87$ & $97.74 \pm 0.85$ \\
\hline \multirow{3}{*}{$\mathrm{Db} 6$} & Level 2 & $98.23 \pm 0.90$ & $98.65 \pm 0.73$ & Coif 2 & Level 2 & $98.10 \pm 0.90$ & $98.69 \pm 0.70$ \\
\hline & Level 4 & $98.48 \pm 0.63$ & $98.53 \pm 0.69$ & & Level 4 & $98.34 \pm 0.79$ & $98.52 \pm 0.66$ \\
\hline & Level 6 & $97.48 \pm 0.88$ & $97.52 \pm 0.85$ & & Level 6 & $97.59 \pm 0.91$ & $97.70 \pm 0.77$ \\
\hline \multirow{3}{*}{$\mathrm{Db} 8$} & Level 2 & $98.20 \pm 0.90$ & $98.69 \pm 0.71$ & Coif 3 & Level 2 & $98.18 \pm 0.90$ & $98.69 \pm 0.71$ \\
\hline & Level 4 & $98.44 \pm 0.67$ & $98.59 \pm 0.67$ & & Level 4 & $98.49 \pm 0.70$ & $98.62 \pm 0.62$ \\
\hline & Level 6 & $97.57 \pm 0.74$ & $97.60 \pm 0.82$ & & Level 6 & $97.56 \pm 0.85$ & $97.71 \pm 0.73$ \\
\hline \multirow{3}{*}{$\mathrm{Db} 10$} & Level 2 & $98.17 \pm 0.94$ & $98.70 \pm 0.68$ & Coif 4 & Level 2 & $98.22 \pm 0.93$ & $98.70 \pm 0.70$ \\
\hline & Level 4 & $98.48 \pm 0.66$ & $98.62 \pm 0.60$ & & Level 4 & $98.42 \pm 0.74$ & $98.56 \pm 0.68$ \\
\hline & Level 6 & $97.43 \pm 0.91$ & $97.49 \pm 0.88$ & & Level 6 & $97.61 \pm 0.83$ & $97.71 \pm 0.72$ \\
\hline \multirow{3}{*}{ Sym 2} & Level 2 & $97.97 \pm 1.01$ & $98.63 \pm 0.68$ & Coif 5 & Level 2 & $98.23 \pm 0.88$ & $98.70 \pm 0.71$ \\
\hline & Level 4 & $98.31 \pm 0.72$ & $98.44 \pm 0.70$ & & Level 4 & $98.45 \pm 0.72$ & $98.56 \pm 0.59$ \\
\hline & Level 6 & $97.32 \pm 0.94$ & $97.45 \pm 0.78$ & & Level 6 & $97.50 \pm 0.86$ & $97.59 \pm 0.85$ \\
\hline
\end{tabular}
Decomposition Level Across Ten Subjects 
In the second part of the experiments, 12 mother wavelets of CWT are studied. Table 3 demonstrates the mean classification accuracy of 12 mother wavelets of CWT at scale 8,16 and 32 for ten different subjects. At scale 8, Gaussian 2 and 4 exhibit the highest classification accuracy of $98.42 \%$ using WL and MAV feature sets, respectively. However, their performance did not show much improvement at a higher scale. At scale 16, it has been found that the Symlet 6 achieves the best classification accuracy of 98.56\%, followed by Symlet 4, 98.53\% when MAV is used. For instance, the Mexican hat has shown its superiority at scale 32 with the best mean classification accuracy of $98.64 \%$ in WL feature set. Unfortunately, MAV shows the decrement in classification performance at scale 32. This shows that MAV feature set is not suitable for high scale wavelet function in CWT. As a result, the most suitable mother wavelet in CWT are Mexican hat at scale 32 and Symlet 6 at scale 16.

Table 3. Classification Accuracy (mean \pm STD) of 12 Mother Wavelets of CWT at Three Different Scale Across Ten Subjects

\begin{tabular}{|c|c|c|c|c|c|c|c|}
\hline \multirow{2}{*}{\multicolumn{2}{|c|}{ Mother wavelet }} & \multicolumn{2}{|c|}{ Classification performance (\%) } & \multirow{2}{*}{\multicolumn{2}{|c|}{ Mother wavelet }} & \multicolumn{2}{|c|}{ Classification performance (\%) } \\
\hline & & WL & MAV & & & WL & MAV \\
\hline \multirow{3}{*}{ Haar } & Scale 8 & $97.70 \pm 0.96$ & $98.00 \pm 1.08$ & \multirow[t]{3}{*}{ Sym 6} & Scale 8 & $98.05 \pm 0.86$ & $98.17 \pm 0.97$ \\
\hline & Scale 16 & $98.38 \pm 0.92$ & $98.31 \pm 0.96$ & & Scale 16 & $98.48 \pm 0.81$ & $98.56 \pm 0.74$ \\
\hline & Scale 32 & $98.51 \pm 0.76$ & $98.19 \pm 0.79$ & & Scale 32 & $98.49 \pm 0.72$ & $98.35 \pm 0.73$ \\
\hline \multirow{3}{*}{$\mathrm{Db} 2$} & Scale 8 & $97.88 \pm 1.01$ & $98.06 \pm 1.13$ & \multirow[t]{3}{*}{ Morl } & Scale 8 & $98.00 \pm 0.83$ & $98.07 \pm 0.83$ \\
\hline & Scale 16 & $98.44 \pm 0.88$ & $98.42 \pm 0.86$ & & Scale 16 & $98.40 \pm 0.86$ & $98.40 \pm 0.82$ \\
\hline & Scale 32 & $98.50 \pm 0.72$ & $98.29 \pm 0.73$ & & Scale 32 & $98.34 \pm 0.74$ & $98.26 \pm 0.78$ \\
\hline \multirow{3}{*}{$\mathrm{Db} 4$} & Scale 8 & $97.99 \pm 0.92$ & $98.13 \pm 1.03$ & \multirow[t]{3}{*}{ Meyr } & Scale 8 & $98.06 \pm 0.95$ & $98.13 \pm 0.95$ \\
\hline & Scale 16 & $98.46 \pm 0.90$ & $98.47 \pm 0.78$ & & Scale 16 & $98.45 \pm 0.84$ & $98.49 \pm 0.75$ \\
\hline & Scale 32 & $98.45 \pm 0.76$ & $98.27 \pm 0.76$ & & Scale 32 & $98.36 \pm 0.79$ & $98.29 \pm 0.81$ \\
\hline \multirow{3}{*}{$\mathrm{Db} 6$} & Scale 8 & $97.94 \pm 1.01$ & $98.08 \pm 1.08$ & \multirow[t]{3}{*}{ Mexh } & Scale 8 & $98.36 \pm 0.82$ & $98.15 \pm 0.79$ \\
\hline & Scale 16 & $98.41 \pm 0.93$ & $98.46 \pm 0.78$ & & Scale 16 & $98.08 \pm 0.76$ & $97.49 \pm 0.81$ \\
\hline & Scale 32 & $98.36 \pm 0.75$ & $98.27 \pm 0.76$ & & Scale 32 & $98.64 \pm 0.66$ & $96.26 \pm 1.00$ \\
\hline \multirow{3}{*}{ Sym 2} & Scale 8 & $97.88 \pm 1.01$ & $98.06 \pm 1.13$ & \multirow[t]{3}{*}{ Gaus 2} & Scale 8 & $98.42 \pm 0.83$ & $98.35 \pm 0.84$ \\
\hline & Scale 16 & $98.44 \pm 0.88$ & $98.42 \pm 0.86$ & & Scale 16 & $98.28 \pm 0.76$ & $98.00 \pm 0.77$ \\
\hline & Scale 32 & $98.50 \pm 0.72$ & $98.29 \pm 0.73$ & & Scale 32 & $98.50 \pm 0.67$ & $97.01 \pm 0.87$ \\
\hline \multirow{3}{*}{ Sym 4} & Scale 8 & $98.03 \pm 0.87$ & $98.18 \pm 0.99$ & \multirow[t]{3}{*}{ Gaus 4} & Scale 8 & $98.39 \pm 0.93$ & $98.42 \pm 0.83$ \\
\hline & Scale 16 & $98.48 \pm 0.83$ & $98.53 \pm 0.74$ & & Scale 16 & $98.48 \pm 0.70$ & $98.42 \pm 0.71$ \\
\hline & Scale 32 & $98.52 \pm 0.69$ & $98.34 \pm 0.74$ & & Scale 32 & $98.31 \pm 0.69$ & $97.80 \pm 0.77$ \\
\hline
\end{tabular}

In the final part of the experiments, the paired two-tail $t$-test is used to measure the statistical difference between the classification performances of WL and MAV features when different mother wavelet function is used. Table 4 and 5 outline the result of $t$-test of the classification performance obtained from DWT and CWT across ten subjects. In $t$-test, the null hypothesis is rejected if the $p$-value is less than 0.05 . This shows that there is a statistical difference between WL and MAV feature sets.

From Table 4, the results of the WL and MAV are statistical difference for all wavelet functions at the second decomposition level. At fourth decomposition level, the $p$-value illustrates that the Daubechies 6 and Coiflet 5 show no significant difference when WL versus MAV. At sixth decomposition level, only Haar, Daubechies 4 and Symlet 4 exhibit the significant difference. From Table 5, Haar, Symlet 4 and Mexican hat show significant difference in scale 8. Additionally, at scale 16, only Mexican hat, Gaussian 2 and Gaussian 4 obtain p-value lower than 0.05. Moreover, other than Daubechies 6 and Symlet 6 exhibit significant differences between the classification performance of WL and MAV at scale 32.

Table 4. Result of $t$-test of the Classification Performance between MAV and WL using DWT

\begin{tabular}{lccc}
\hline Mother wavelet & Level 2 & $\begin{array}{c}p-\text { value } \\
\text { Level 4 }\end{array}$ & Level 6 \\
\hline Haar & 0.0007 & 0.0007 & $3 \mathrm{E}-05$ \\
Db 2 & 0.0012 & 0.0195 & 0.0521 \\
$\mathrm{Db} 4$ & 0.0006 & 0.0087 & 0.0031 \\
$\mathrm{Db} 6$ & 0.0070 & 0.3754 & 0.2340 \\
Db 8 & 0.0037 & 0.0185 & 0.6085 \\
Db 10 & 0.0020 & 0.0036 & 0.3163 \\
Sym 2 & 0.0012 & 0.0195 & 0.0521 \\
Sym 4 & 0.0009 & 0.0057 & 0.0138 \\
Sym 6 & 0.0008 & 0.0046 & 0.0380 \\
Sym 8 & 0.0007 & 0.0289 & 0.1081 \\
Coif 2 & 0.0012 & 0.0178 & 0.0854 \\
Coif 3 & 0.0031 & 0.0109 & 0.0625 \\
Coif 4 & 0.0031 & 0.0157 & 0.0504 \\
Coif 5 & 0.0010 & 0.0860 & 0.0807 \\
\hline
\end{tabular}


Table 5. Result of $t$-test of the Classification Performance between MAV and WL using CWT.

\begin{tabular}{lccc}
\hline Mother wavelet & Scale 8 & $\begin{array}{c}p-\text { value } \\
\text { Scale 16 }\end{array}$ & Scale 32 \\
\hline Haar & 0.0377 & 0.2676 & 0.0003 \\
Db 2 & 0.0539 & 0.8141 & 0.0003 \\
Db 4 & 0.1104 & 0.8478 & 0.0104 \\
Db 6 & 0.0525 & 0.3855 & 0.0670 \\
Sym 2 & 0.0539 & 0.8141 & 0.0003 \\
Sym 4 & 0.0409 & 0.5256 & 0.0037 \\
Sym 6 & 0.0625 & 0.2172 & 0.0526 \\
Morl & 0.0635 & 0.9162 & 0.0050 \\
Meyr & 0.1266 & 0.3865 & 0.0207 \\
Mexh & $3 \mathrm{E}-05$ & $7 \mathrm{E}-07$ & $2 \mathrm{E}-06$ \\
Gaus 2 & 0.2864 & $7 \mathrm{E}-05$ & $7 \mathrm{E}-07$ \\
Gaus 4 & 0.5683 & 0.0334 & $5 \mathrm{E}-05$ \\
\hline
\end{tabular}

\section{CONCLUSION}

In this study, the usefulness of the mother wavelet function in DWT and CWT has been investigated. Two popular features, WL and MAV are extracted from the wavelet coefficients as the input to the SVM. In CWT, the Mexican hat at scale 32 and Symlet 6 at scale 16 are suggested to be the optimal mother wavelet selection for the classification of EMG signals. On the other hand, the reconstructed DWT coefficient with Daubechies 4 and Symlet 4 at second decomposition level are recommended to be used in EMG pattern recognition. The experimental results indicated DWT not only offered low computation cost, but also yielded a high classification accuracy. As compared to CWT, DWT is more approariate to be used in rehabilitation and clinical application.

\section{ACKNOWLEDGEMENTS}

The authors would like to thank the Universiti Teknikal Malaysia Melaka (UTeM), Skim Zamalah UTeM and Minister of Higher Education Malaysia (MOHE) for funding research under grant PJP/1/2017/FKEKK/H19/S01526.

\section{REFERENCES}

[1] A. Phinyomark, et al., "Feature reduction and selection for EMG signal classification," Expert System with Application, vol/issue: 39(8), pp. 7420-7431, 2012.

[2] G. Vannozzi, et al., "Automatic detection of surface EMG activation timing using a wavelet transform based method," Journal of Electromyography and Kinesiology, vol/issue: 20(4), pp. 767-772, 2010.

[3] A. Phinyomark, et al., "Application of Wavelet Analysis in EMG Feature Extraction for Pattern Classification," Measurement Science Review, vol/issue: 11(2), pp. 45-52, 2011.

[4] A. C. Tsai, et al., "A novel STFT-ranking feature of multi-channel EMG for motion pattern recognition," Expert System with Application, vol/issue: 42(7), pp. 3327-3341, 2015.

[5] R. H. Chowdhury, et al., "Surface Electromyography Signal Processing and Classification Techniques," Sensors, vol/issue: 13(9), pp. 12431-12466, 2013.

[6] F. A. Omari, et al., "Pattern Recognition of Eight Hand Motions Using Feature Extraction of Forearm EMG Signal," Proceedings of the National Academy of Sciences, India Section A: Physical Sciences, vol/issue: 84(3), pp. 473-480, 2014.

[7] M. R. Canal, "Comparison of Wavelet and Short Time Fourier Transform Methods in the Analysis of EMG Signals," Journal of medical systems, vol/issue: 34(1), pp. 91-94, 2010.

[8] N. M. Kakoty, et al., "Exploring a family of wavelet transforms for EMG-based grasp recognition," Signal, Image and Video Processing, vol/issue: 9(3), pp. 553-559, 2015.

[9] J. Rafiee, et al., "Wavelet basis functions in biomedical signal processing," Expert System with Application, vol/issue: 38(5), pp. 6190-6201, 2011.

[10] M. Saini, et al., "Algorithm for Fault Location and Classification on Parallel Transmission Line using Wavelet based on Clarke's Transformation," International Journal of Electrical and Compututer Engineering. IJECE, vol/issue: 8(2), pp. 699-710, 2018.

[11] A. Phinyomark, et al., "Feature Extraction and Reduction of Wavelet Transform Coefficients for EMG Pattern Classification," Elektronika ir Elektrotechnika, vol/issue: 122(6), pp. 27-32, 2012.

[12] J. Yousefi and A. H. Wright, "Characterizing EMG data using machine-learning tools," Computer in Biology and Medicine, vol. 51, pp. 1-13, 2014.

[13] L. H. Smith, et al., "Determining the Optimal Window Length for Pattern Recognition-Based Myoelectric Control: Balancing the Competing Effects of Classification Error and Controller Delay," IEEE Transactions on Neural Systems and Rehabilitation Engineering, vol/issue: 19(2), pp. 186-192, 2011. 
[14] M. Hakonen, et al., "Current state of digital signal processing in myoelectric interfaces and related applications," Biomedical Signal Processing and Control, vol. 18, pp. 334-359, 2015.

[15] J. Rafiee, et al., "Feature extraction of forearm EMG signals for prosthetics," Expert System with Application, vol/issue: 38(4), pp. 4058-4067, 2011.

[16] L. Fraiwan, et al., "Automated sleep stage identification system based on time-frequency analysis of a single EEG channel and random forest classifier," Computer methods and programs in biomedicine, vol/issue: 108(1), pp. 1019, 2012.

[17] S. H. Cho, et al., "Time-Frequency Analysis of Power-Quality Disturbances via the Gabor Wigner Transform," IEEE transactions on power delivery, vol/issue: 25(1), pp. 494-499, 2010.

[18] A. Subasi, "Classification of EMG signals using PSO optimized SVM for diagnosis of neuromuscular disorders," Comput. Biol. Med., vol/issue: 43(5), pp. 576-586, 2013.

[19] M. H. D. Mohammadi, "Improved Denoising Method for Ultrasonic Echo with Mother Wavelet Optimization and Best-Basis Selection,” International Journal of Electrical and Compututer Engineering. IJECE, vol/issue: 6(6), pp. 2742-2754, 2016.

[20] A. Subasi, "Classification of EMG signals using combined features and soft computing techniques," Applied Soft Computing, vol/issue: 12(8), pp. 2188-2198, 2012.

[21] S. V. S. Prasad, et al., "Comparison of Accuracy Measures for RS Image Classification using SVM and ANN Classifiers," International Journal of Electrical and Compututer Engineering. IJECE, vol/issue: 7(3), pp. 11801187, 2017.

[22] A. Subasi and M. I. Gursoy, "EEG signal classification using PCA, ICA, LDA and support vector machines," Expert System with Application, vol/issue: 37(12), pp. 8659-8666, 2010.

\section{BIOGRAPHIES OF AUTHORS}
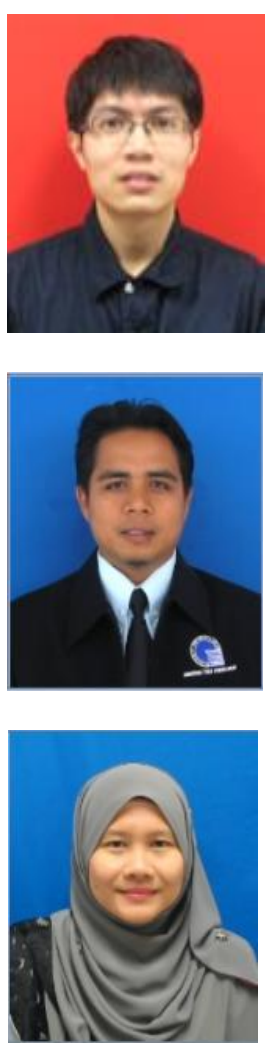

Too Jing Wei has received his B. Eng. from Universiti Teknikal Malaysia in 2017. He is currently pursuing his Master Eng. in Universiti Teknikal Malaysia. His research areas are in signal processing, classification and feature selection for EMG pattern recognition.

Associate Prof. Dr. Abdul Rahim Bin Abdullah has received his B. Eng., Master Eng., PhD Degree from Universiti Teknologi Malaysia in 2001, 2004 and 2011 in Electrical Engineering and Digital Signal Processing respectively. He is currently an Associate Professor with the Department of Electrical Engineering for Universiti Teknikal Malaysia Melaka (UTeM).

Dr. Norhashimah Binti Mohd Saad is currently working as a senior lecturer in Department Computer, FKEKK, UTeM. She finished her study in Bachelor of Engineering, Master of Engineering and $\mathrm{PhD}$ in Medical Image Processing from UTM, Malaysia. 